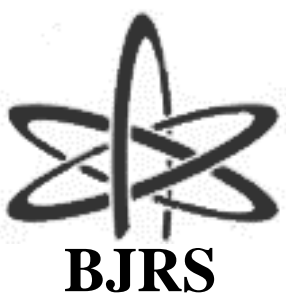
BRAZILIAN JOURNAL
$\mathrm{OF}$
RADIATION SCIENCES
06-01 (2018) 01-13

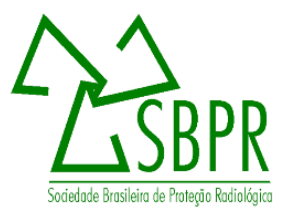

\title{
Construindo pontes entre ciência e sociedade: divulgação científica sobre irradiação de alimentos
}

\author{
D. S. Levy ${ }^{\text {ab; G. M. A. A. Sordi }}$; A. L. C. H. Villavicencio ${ }^{\mathrm{a}}$ \\ ${ }^{a}$ Cenro de Tecnologia das Radiações, Instituto de Pesquisas Energéticas e Nucleares, São Paulo, Brasil \\ ${ }^{b}$ Gerência de Radioproteção, Instituto de Pesquisas Energéticas e Nucleares, São Paulo, Brasil \\ denise@omiccron.com.br
}

\section{RESUMO}

A irradiação de alimentos é uma técnica de preservação segura e eficaz para eliminar insetos e reduzir microrganismos prejudiciais à saúde humana. O processo contribui para inibir o brotamento e retardar o amadurecimento dos alimentos, prolongando a vida útil de frutas e legumes frescos. Entretanto, dentre uma grande parcela da população brasileira, ainda há grande desinformação sobre as aplicações pacíficas das ciências nucleares. Não raramente a mídia e as redes sociais tendem a associar a radiação ionizante a armas nucleares e acidentes de grandes proporções. A desinformação compromete a percepção do público: consumidores acabam por associar alimentos irradiados a alimentos radioativos ou contaminados. O público demonstra grandes preocupações quanto aos efeitos negativos das radiações para a saúde e riscos de contaminação ambiental. Os julgamentos e tomadas de decisões por parte da sociedade estão diretamente ligados à sua percepção dos riscos e benefícios advindos da Tecnologa Nuclear. Este artigo apresenta algumas considerações sobre as percepções do público a respeito dos alimentos irradiados, bem como algumas ações de divulgação científica, para aproximar ciência e sociedade. Cabe à comunidade científica construir novas pontes entre as ciências nucleares e o público leigo, oferecendo uma perspectiva mais ampla sobre as aplicações pacíficas das radiações ionizantes e suas contribuições na vida cotidiana.

Palavras-chave: Irradiação de alimentos, percepção de risco, aceitação pública. 


\begin{abstract}
Food irradiation is a safe and effective preservation technique to eliminate insects and parasites and reduce diseasecausing microorganisms. The process helps to inhibit sprouting and delay ripening, prolonging the shelf-life of fresh fruits and vegetables. Nevertheless, among a great fraction of Brazilian population, there is still great misunderstanding about the peaceful applications of nuclear science. Most often the media and social networks tend to associate radiation to nuclear weapons and major accidents. Misinformation and common wrong beliefs confuse public's perceptions: consumers seem to misunderstand the difference between irradiated food and radioactive food and the general public has major concerns about the negative health effects and environmental contamination. This paper presents some considerations about consumers' perceptions of irradiated food, as well as the need of improving science communication. Society's judgments and decision making are directly linked to perceived benefits and risks. The scientific community must build new bridges between nuclear science and the general public, offering a wider perspective on the benefits and risks resulting from ionizing radiation in everyday life.
\end{abstract}

Keywords: Food irradiation, risk perception, consumers acceptance.

\title{
1. INTRODUÇÃO
}

A temática da alimentação é uma das grandes questões brasileiras e a irradiação de alimentos é um dos campos de pesquisa que desponta como uma importante contribuição para responder à emergência desta demanda. A irradiação de alimentos é uma técnica eficaz e ambientalmente segura, que contribui para dois aspectos primordiais: a segurança do alimento e o combate ao desperdício. O processo auxilia na conservação e desinfecção dos alimentos, eliminando insetos e reduzindo significativamente a carga microbiana e organismos patogênicos à saúde humana. Ainda, o processo de irradiação de alimentos previne o brotamento, retardando o amadurecimento de frutas, hortaliças e legumes, prolongando o tempo de vida útil dos alimentos. Com efeito, as radiações ionizantes estão presentes no nosso cotidiano e contribuem para melhorar a qualidade de vida da população. Entretanto, o tema ainda divide a opinião pública e, dentre grande parte da população, ainda é grande a desinformação sobre as aplicações pacificas das ciências nucleares. Não raramente a mídia e as redes sociais relacionam Tecnologia Nuclear a armas e enfatizam de grandes proporções. Os julgamentos por parte da população estão diretamente ligados às informações disponíveis e não é coincidência que pessoas mais 
informadas tendem a favorecer a tecnologia nuclear. Importantes pesquisas conduzidas pela GlobeScan (2005) e Eurobarometers (2010) relatam tendências sócio-demográficas semelhantes: quanto maior o grau de instrução, mais favorável a opinião pública. Eurobarometers são utilizados para medir a opinião pública na Europa e no exterior. O relatório, intitulado "Atitudes públicas de Energia Nuclear", foi publicado em 2010 pela Agência de Energia Nuclear (AEN) e a Organização para a Cooperação Econômica e Desenvolvimento (OCDE). Segundo este documento, "pessoas com maior nível de escolaridade são mais propensas a pensar que as vantagens da energia nuclear superam os riscos". [1]

A pesquisa Globescan (2005) para a Agência Internacional de Energia Atômica (AIEA), publicada em 2005, analisa os resultados de opinião pública entre mil adultos entrevistados em cada um dos dezoito países participantes [2]. De acordo com este relatório:

"No geral, os homens (33\%) e pessoas com altos níveis de educação (36\%) são mais propensos do que as mulheres (23\%) e aqueles com baixos níveis de educação (24\%) para alegar que a energia nuclear é segura e que os países interessados devem construir novas usinas nucleares. Pessoas com menor escolaridade (28\%) são mais propensas do que as bem-informadas (21\%) para dizer que a energia nuclear é perigosa e que todas as plantas devem ser fechadas."

No entanto, uma pesquisa realizada pela Globescan (2011) para a BBC World Service após a crise de Fukushima traz tendências diferentes: a oposição à energia nuclear cresce desde 2005 [3]. A pesquisa abrangeu 23.231 cidadãos em 23 países. O Brasil foi um dos países participantes deste estudo, embora as respostas reflitam apenas a opinião da população urbana:

"No Brasil - que opera algumas usinas nucleares e foi entrevistado pela primeira vez em 2011 - uma pluralidade de 44 por cento dos brasileiros diz que seu país deve continuar a usar as usinas nucleares que já estão em operação, mas não deve construir novas usinas. Trinta e cinco por cento diz que a energia nuclear é perigosa e que todas as usinas nucleares em operação devem ser fechadas o mais rápidamente possível - acima da média de 12 países (30\%) - e apenas 16 por cento apoia a construção de novas usinas nucleares - abaixo da média de 12 países (22\%) ". [3]

Em 2009, foi realizado um estudo para medir a aceitação do público brasileiro em relação à irradiação de alimentos. As pesquisas mostraram que o público não sabia a diferença entre 
"alimentos irradiados" e "alimentos radioativos" e as pessoas temiam os efeitos nocivos das radiações ionizantes para a saúde dos consumidores. [4]

Em novembro de 2016, os autores do presente artigo empreenderam uma pesquisa para avaliar o conhecimento prévio de consumidores em relação à irradiação de alimentos. $50 \%$ dos entrevistados eram professores com nível universitário. Os resultados demonstraram o completo desconhecimento sobre o tema por grande parte dos entrevistados.

A divulgação científica é um desafio e uma necessidade. O objetivo do presente artigo é discutir possibilidades viáveis para a construção novas pontes entre as ciências nucleares e o público leigo, oferecendo perspectivas mais amplas sobre as aplicações pacíficas das radiações ionizantes e suas contribuições na vida cotidiana. O artigo apresenta ainda o desenvolvimento de um projeo de divulgação científica, incluindo pesquisa junto ao público, estudos sobre as Tecnologias de Informação e Comunicação e o desenvolvimento de um conteúdo programático capaz de atender aos interesses plurais dos diversos setores da sociedade.

\section{MATERIAIS E MÉTODOS}

O presente trabalho descreve a evolução de uma proposta de tecnologia educacional para a disseminação da informação. O desenvolvimento deste projeto envolveu três etapas distintas:

- Pesquisa junto ao público.

- Pesquisa sobre evolução das Tecnologias da Informação e Comunicação no Brasil

- Desenvolvimento de conteúdo.

\subsection{PESQUISA JUNTO AO PÚBLICO}

Em novembro de 2016, os autores do presente artigo empreenderam uma pesquisa presencial, para a qual foram convidados 100 entrevistados residentes na capital paulista, incluindo educadores (50\% dos entrevistados) e membros da socedade em geral: estudantes, empresários, donas de casa e profissionais pertencentes aos mais diversos ramos. Quando perguntados sobre as primeiras ideias que lhes vinham em mente quando pensavam em tecnologia Nuclear, 55\% dos entrevistados associou radiações ionizantes a guerras, contaminação, acidentes nucleares, doenças, cânceres ou mortes (ver Figura 1). Para a questão "você já experimentou alimentos irradiados?", as respostas mais cocmuns foram: (1) sim, porém sem 
saber que os alimentos foram irradiados, ou (2) sim, porque grande parte do alimento que comsumimos provém de solos contaminados. Quando lhes foi apresentado o símbolo de Radura, 67\% dos entrevistados alegaram nunca tê-lo visto; apenas $21 \%$ alegaram reconhecer o símbolo, porém não sabiam o que representava. Os entrevistados foram, em seguida, informados que a Radura é o símbolo internacionalmente utilizado para identificação de produtos alimentares irradiados, após o que lhes foi perguntado se eles, conhecendo o símbolo, escolheriam alimentos que contivessem a radura. $29 \%$ dos entrevistados responderam que não; $18 \%$ alegaram precisar de mais informações a respeito, $6 \%$ responderam que sim, pois associaram o símbolo a um selo de qualidade (ver Figura 2). Ao final da entrevista, os entrevistados receberam uma brevíssima explicação sobre esta técnica de preservação para eliminar insetos e reduzir microrganismos causadores de doenças. Foi-lhes igualmente explicada a diferença entre "alimentos irradiados" e "alimentos contaminados". Após a explicação, os entrevistados foram convidados a responder novamente sobre suas intenções de compra de alimentos irradiados. As opiniões dos entrevistados mudaram significativamente: $70 \%$ respondeu que sim, escolheria, informaria a amigos e parentes e até sugerira a compra para outras pessoas. $16 \%$ afirmou que sim, escolheria, desde que obtivesse maiores informações sobre o processo e os efeitos no longo prazo para a saúde e o impactos que no meio ambiente.

Figura 1: Palavras ou expressões mais frequentes

(Fonte: Elaborada pelos autores. Acervo pessoal)

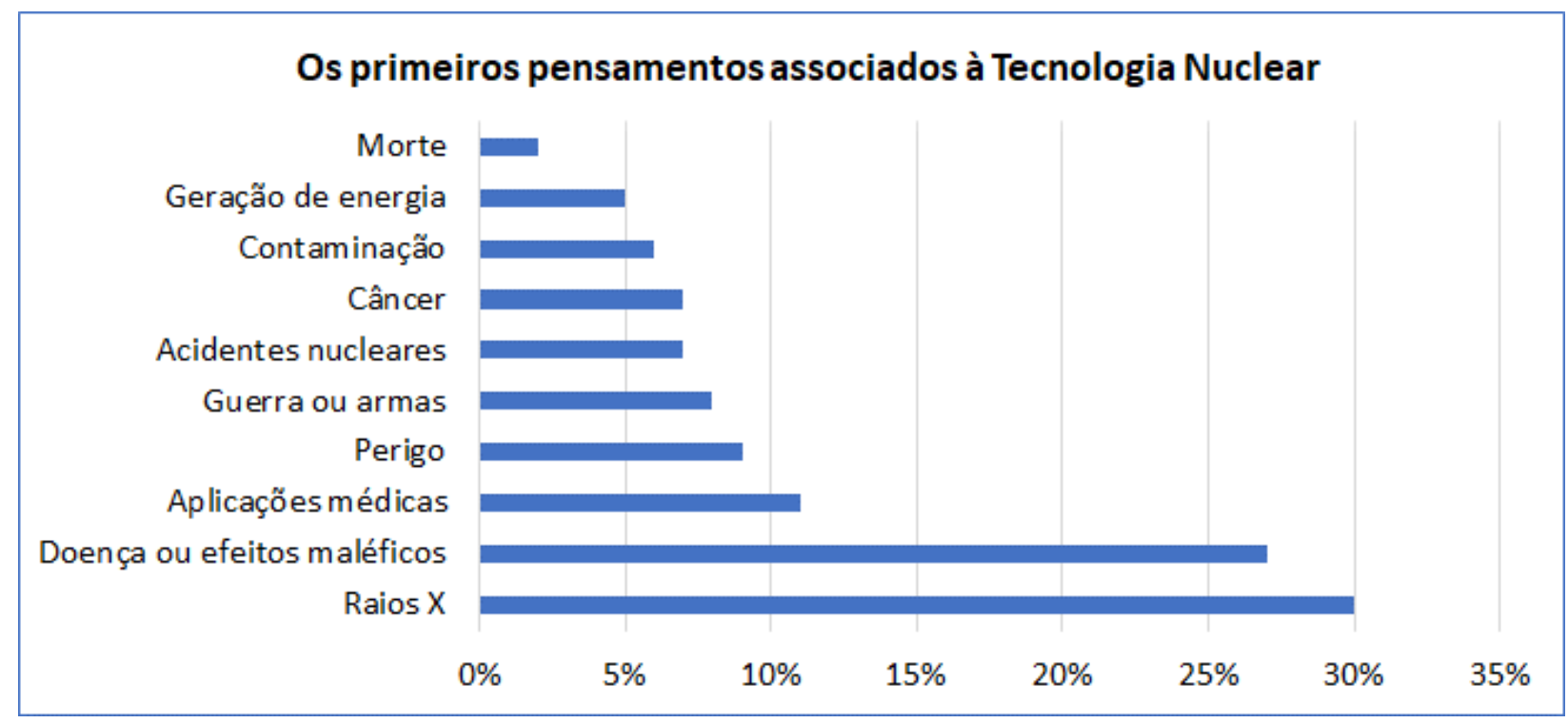


Figura 2: Intenção de compra dos consumidores antes de receberem informação

(Fonte: Elaborada pelos autores. Acervo pessoal)

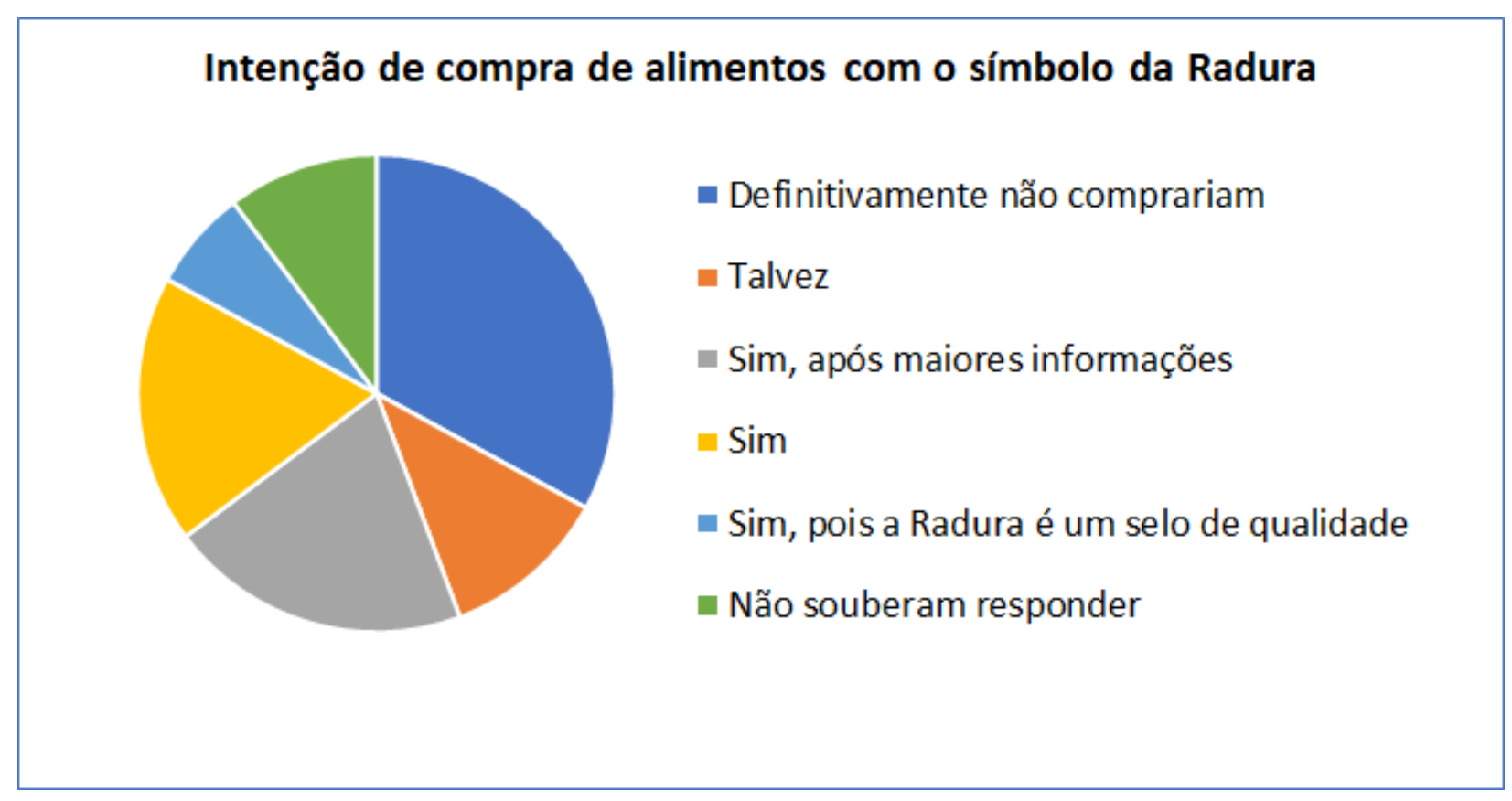

\subsection{ACESSO ÀS TECNOLOGIAS DA INFORMAÇÃO E COMUNICAÇÃO}

A equipe de pesquisadores acompanha anulamente as publicações oficiais quanto às tendências de acesso à TICs em todo o país, nos domicílios e escolas. Em 2015, publicações do Comitê Gestor da Internet no Brasil [5 - 8] indicaram avanços significativos na utilização das TICs em relação aos anos precedentes. A pesquisa TIC Domicilios [5] tem por objetivo "subsidiar a sociedade com dados oficiais sobre os impactos socioeconômicos das TICs, contribuindo para políticas públicas mais efetivas e eficazes e para o desenvolvimento da Internet no Brasil". Dentre os 65.129.753 domicílios que responderam à pesquisa, em todos os estados brasileiros, $92 \%$ dos entrevistados declararam possuir telefone celular para acesso à Internet. 50\% declararam possuir computadores em casa. Dentre os 32.881 .928 domicílios que possuem computador, $56 \%$ declararam possuir computador de mesa, $60 \%$ possuem comutador portátil e $33 \%$ possuem tablets. $39 \%$ possui mais de um computador. A pesquisa revelou que possuem computadores : $98 \%$ das famílias da classe $\mathrm{A} ; 82 \%$ das famílias pertencentes à classe $\mathrm{B} ; 48 \%$ de famílias da classe $\mathrm{C}$ e $14 \%$ de famílias da classe D. A grande maioria conta com acesso de banda larga; apenas $2 \%$ dos entrevistados possuem acesso discado e $25 \%$ contam com banda larga móvel (3G). 17\% alegam possuir 
conexão entre 256 Kbps e 1 Mbps; $13 \%$ entre 1 Mbps e 2 Mbps; $9 \%$ entre 2 Mbps e 4 Mbps; $8 \%$ entre 4 Mbps e 8 Mbps. 23\% do total de domicílios que têm computadores têm velocidade de conexão acima de 8 Mbps. $66 \%$ das famílias possuem acesso wifi. A pesquisa traz igualmente dados sobre a proporção de usuários de Internet por atividades realizadas na busca de informações: 47\% dos entrevistados declarou utilizar a Internet para fins de pesquisa acadêmica e informação; 38\% buscam informações em site de enciclopédia; $33 \%$ estudam na Internet por conta própria e 11\% buscam cursos a distância. Em relação ao dispositivo utilizado para acesso individual, 76\% dos usuários declaram utilizar o celular. [6]

Em relação ao acesso às TICs nas escolas brasileiras, incluindo escolas públicas e privadas, os resultados mostram que $97 \%$ delas utilizam recursos da Internet para angariar conteúdo a ser usado em aula. Os professores se consideraram capacitados para o uso de recursos digitais e alegam fazer uso das TICs em suas práticas de ensino. Uma significativa parcela dos professores procura trabalhar conteúdos que envolvam a participação ativa dos estudantes. No entanto, foram relatados problemas relacionados à qualidade de acesso à Internet nas escolas públicas, devido à baixa velocidade de conexão. Quanto ao acesso dos alunos à Internet, a edição de 2014 da pesquisa registrou um aumento importante no uso da Internet por meio de telefones celulares. Em 2013, apenas 59\% desses estudantes usavam telefones celulares para acessar a Internet. Em 2014, 79\% dos alunos alegaramm fazer uso dos celulares para acessar conteúdos na Internet, dentre os quais $75 \%$ dos estudantes pesquisados declararam utilizar as TICs para a realização de tarefas de casa, exercícios e pesquisas. [8]

Em posse destes dados, deu-se início ao desenvolvimento de modelos de tecnologia educacional para disseminação do conteúdo de irradiação de alimentos. O desafio envolveu a capacidade de criar uma comunicação eficaz que atenda aos interesses plurais dos diversos segmentos da sociedade, utiizando ferramentas e recursos que permitem a adequada estrutura organizacional e que atenda às possibilidades de acesso por parte do público alvo.

\section{RESULTADOS E DISCUSSÕES}

A pesquisa junto ao público demonstou que a tecnologia nuclear ainda é um tema controverso dentre uma grande parcela da população brasileira e a questão da irradiação de alimentos é muitas vezes mal interpretada. É preciso promover a educação da população e a aceitação pública da irradiação de alimentos, desmistificando preconceitos e informando o público consumidor. A introdução da Irradiação de Alimentos no Brasil é uma realidade desde o final 
dos anos 60. Durante as décadas posteriores, houve uma evolução juntamente com o apelo desta tecnologia no mundo. A legislação Brasileira teve seu inicio na ANVISA (Agencia Nacional de vigilância Sanitária, Ministério da Saúde) pelo Decreto-lei no 72.718 de 2 de agosto de 1973. Atualmente vigora a RDC n²1, de 26 de janeiro de 2001. Nesta resolução são descritas as condições da embalagem, o procedimento de irradiação apropriado e a exigência da rotulagem com os dizeres: “ALIMENTO TRATADO POR PROCESSO DE IRRADIAÇÃO”, entre outras exigências. Existe ainda um símbolo internacional (RADURA) para ser utilizado nos produtos alimentícios processados por radiação. O MAPA (Ministério da agricultura, Pecuária e Abastecimento) também tem normativas a respeito da aplicação da radiação em produtos agrícolas (NINF 18). Desde 1974 o IPEN tem equipamentos de irradiação tanto de ${ }^{60}$ Co como de acelerador de elétrons, desenvolvendo e assessorando produtores com pesquisas e testes de seus produtos em uma escala semi-industrial em alimentos e produtos agrícolas, bem como um grupo de pesquisadores atuantes neste tema. O comércio de produtos alimentícios está em amplo desenvolvimento exigindo métodos cada vez mais saudáveis que deixem o alimento com o máximo de suas características e com mínimo ou nenhum dano em seus biocompostos naturais. Neste aspecto o processamento por radiação vem crescendo no Brasil e no mundo, conforme as exigências do mercado consumidor. Consideráveis quantidades de frutos do mar congelados, carnes de diferentes tipos, vegetais minimamente processados, especiarias e frutas frescas e desidratadas são exemplos onde se aplicam a radiação, no sentido de se fornecer e aumentar a segurança dos alimentos são comercializados ou exportados pelo Brasil e não têm rotulagem quando comercializados no mercado consumidor final. No Brasil os irradiadores comerciais estão atualmente sob o comando de uma multinacional Canadense (Sterigenics). Em 1980 a EMBRARAD São Paulo, iniciou junto a suas operações para diversos ramos da indústria da área médica e farmacêutica, a irradiação de alimentos e produtos para a agroindústria de alimentos. Em 1999, inaugurou seu segundo irradiador. Outra indústria de irradiação se estabelece em São Paulo em 1999, a CBE (Companhia Brasileira de Esterilização) com um irradiador de Co60 construído com tecnologia brasileira, também com a mesma finalidade da EMBRARAD. Em 2002, iniciam a irradiação comercial com aceleradores de elétrons. Em 2015 todos irradiadores comerciais de alimentos são comprados pela Sterigenics, que é atualmente uma multinacional com o monopólio da irradiação de alimentos no Brasil. Outros estados brasileiros enviam seus 
produtos até as fabricas de São Paulo, mas ainda não há nenhum concorrente oficializado no país. Outros grupos de investidores estão atualmente interessados e iniciando suas pesquisas de mercado para este segmento, mas ainda estão em fase inicial.

\subsection{EDUCAÇÃO E COMUNICAÇÃO: POSSIBILDADES E LIMITAÇÕES}

Ainda que o currículo nacional brasileiro contemple questões relacionadas às ciências nucleares no Ensino Fundamental 2 e Ensino Médio, o tema não constitui uma disciplina específica, sendo ensinado como parte do ensino da Física, da Química ou da Biologia. De acordo com a Lei Brasileira de Diretrizes e Bases para Educação Nacional (1996) [9] e as Diretrizes Curriculares Nacionais para Educação Básica (2013) [10], as escolas são responsáveis por fornecer o currículo mínimo aos alunos para permitir o desenvolvimento de habilidades e competências para uma compreensão justa sobre reações nucleares e suas aplicações. No entanto, os próprios professores não foram preparados para discutir o tema com a profundidade e, no mais das vezes o tema é abordado de forma reducionaista a fim de cumprir o conteúdo mínimo exigido. $\mathrm{O}$ fato de importantes conceitos de ciências nucleares permanecerem em um nível superficial não é sem consequências: preconceitos infundados e desinformação levam o público a associar técnicas nucleares a acidentes, efeitos nocivos para a saúde ou contaminação ambiental. Para além do conteúdo escolar mínimo exigido, é preciso promover o letramento científico, preparando os alunos para o desenvolvimento de um pensamento crítico mais aprofundado, capacitando-os assim para discutir ciência relacionada às questões reais da vida cotidiana [11]. Cabe à comunidade científica promover o diálogo entre o discurso da ciência e a população. As pesquisas realizadas pelo Comitê Gestor da Internet no Brasil [5 - 8] corroboram que as atuais tendências das Tecnologias da Informação e Comunicação (TICs) vão ao encontro das aspirações de uma sociedade globalizada, plural, inclusiva e participativa, que tem por principais características a disseminação do conhecimento e a democratização da educação. Os dados fornecidos permitiram ao grupo de trabalho desenvolver a estratégia de divulgação científica, escolhendo uma variedade de recursos e funcionalidades que atendam às necessidades do público-alvo com quantidade e qualidade de informação.

O material foi dividido em 8 diferentes módulos, de forma a apresentar as diversas aplicações das técnicas nucleares que contribuem de muitas maneiras para melhorar a segurança do 
alimento, da agricultura à mesa do consumidor. Até o presente momento, foram produzidos quatro materiais para divulgação científica: fundamentos da irradiação de alimentos, segurança alimentar, proteção radiológica e técnicas nucleares para a agricultura. Ainda, há outros quato módulos em desenvolvimento: um breve histórico de irradiação de alimentos, leis e regulamentos para a irradiação de alimentos, análise sensorial de alimentos irradiados e irradiação de flores comestíveis. Todo o conteúdo poderá ser acessado facilmente a partir de qualquer ponto de Internet convencional, com bom desempenho mesmo sem alta velocidade de conexão. [12]

\subsection{PERCEPÇÃO DE RISCO E ACEITAÇÃO DO RISCO}

É importante lembrar que a percepção de risco por parte da sociedade difere da percepção de risco da comunidade científica. Os membros do público temem o que não podem compreender e a intenção de compra de alimentos irradiados esbarra em julgamentos de risco intuitivos dos consumidores e uma percepção de risco errônea sobre os malefícios da tecnologia nuclear para a saúde e o meio ambiente [13]. Por esta razão, além dos aspectos gerais e específicos sobre irradiação de alimentos e segurança do alimento, o material interativo aborda também questões básicas de proteção radiológica, apresentando técnicas e equipamentos utilizados, cuidados com proteção ao trabalhador e ao meio ambiente e controle de qualidade. O público desconhece as questões de proteção radiológica associadas ao uso de radiação ionizante em todas as aplicações industriais, como é o caso da irradiação de alimentos. A proteção radiológica é um campo específico que estuda os efeitos biológicos no corpo humano e estabelece limites de dose compatíveis com outros riscos na vida cotidiana. O limite de dose para a população é de apenas 1 $\mathrm{mSva}^{-1}$. Para os trabalhadores envolvidos os limites de radiação ionizante são muito maiores: um limite anual de $20 \mathrm{mSv}$ (média em 5 anos) ou até $50 \mathrm{mSv}$ em um único ano. A técnica de irradiação de alimentos segue as recomendações e requisitos nacionais e internacionais para garantir a segurança das radiações e a segurança física das fontes radioativas, dos trabalhadores e do meio ambiente.

As TICs devem impactar positivamente na educação científica, envolvendo a sociedade em questões relacionadas ao impacto da ciência nuclear em contextos sociais, tecnológicos, econômicos e políticos. $\mathrm{O}$ acesso à informação é um direito de cidadania fundamental. $\mathrm{O}$ 
impacto crescente das TIC promove oportunidades de aprendizagem inclusivas e democráticas. Espera-se que a divulgação científica estimule o questionamento reconstrutivo, desenvolvendo consciência crítica e confiança para analisar situações, ressignificar informações de mídia e construir suas próprias opiniões, preparando as novas gerações para atuarem como cidadãos ativos em questões de interesse da sociedade. Este projeto educacional busca informar a sociedade, promovendo o engajamento dinâmico com os consumidores que parecem temer os efeitos nocivos das radiações ionizantes para a saúde humana e o meio ambiente.

\section{CONSIDERAÇÕES FINAIS}

O presente artigo apresenta algumas considerações sobre a percepção dos consumidores brasileiros de alimentos irradiados, bem como a necessidade de melhorar a comunicação científica. Os julgamentos dos consumidores e a tomada de decisões dependem da sua percepção sobre riscos e benefícios. Cabe à comunidade científica construir novas pontes entre a ciência nuclear e o público leigo, oferecendo uma perspectiva mais ampla e cientificamente correta das aplicações benéficas das radiações ionizantes na vida cotidiana.

A irradiação de alimentos contribui para a segurança alimentar, reduzindo microrganismos e parasitas patogênicos, além de contribuir para o prolongamento da vida útil dos alimentos, reduzindo o desperdício de alimentos. A irradiação de alimentos traz ainda benefícios para a economia do país, tornando o país competitivo no mercado externo. O Brasil é um grande produtor e exportador de alimentos e o processo de irradiação é uma solução viável para a eliminação de insetos considerados pragas quarentenárias. Apesar de todos esses benefícios, nota-se claramente que a aceitação pública é negativa e a população, no mais das vezes, desinformada. [13]. Alguns fabricantes que utilizam alimentos irradiados evitam a palavra "irradiação" que pode desencorajar o consumidor, substituindo por dizeres outros, como por exemplo: "esterilização a frio". A educação e a comunicação são essenciais para aumentar o diálogo entre ciência e público em geral . Acreditamos que esta iniciativa educacional baseada na Internet irá fornecer informações confiáveis e acessíveis, melhorar a compreensão pública da tecnologia nuclear, desmistificar paradigmas e combater a desinformação e os preconceitos 
infundados sobre as aplicações benéficas da tecnologia nuclear em nossas vidas cotidianas. Em um grande país como o Brasil, com regiões geograficamente distantes, esta iniciativa pode contribuir para fornecer informações a populações geograficamente distantes, com baixos custos e alta qualidade. Este é um projeto de longo prazo e é nosso objetivo torná-lo uma referência para todos os países de língua portuguesa.

\section{AGRADECIMENTO}

Os autores agradecem ao Instituto de Pesquisas Energéticas e Nucleares, IPEN-CNEN/SP, que possibilitou a realização do presente trabalho. Agradecemos ainda à Fundação de Amparo à Pesquisa do Estado de São Paulo (FAPESP) e ao Conselho Nacional de Desenvolvimento Científico e Tecnológico (CNPq) pelas valiosas contribuições ao desenvolvimento deste projeto.

\section{REFERÊNCIAS}

1. Nuclear Energy Agency; Organization for Economic Co-operation and development, Public Attitudes to Nuclear Power. Available at: http://www.oecdnea.org/ndd/reports/2010/nea6859-public-attitudes.pdf (2010) Last accessed in December 2016.

2. International Atomic Energy Agency, Global Public Opinion on Nuclear Issues and the IAEA Final Report from 18 Countries, http://large.stanford.edu/courses/2015/ph241/llanos1/docs/globescan.pdf (2005) Last accessed in December 2016.

3. BBC World Service, Opposition to Nuclear Energy Grows: Global Poll. Available at: http://www.globescan. com/images/images /pressreleases/ bbc2011_ nuclear_energy/bbc2011_energy.pdf (2011) Last accessed in December 2016.

4. Behrens, J. H.; Barcellos, M.N.; L. J.; Nunes, T. P.; Landgraf, M. Brazilian consumer views on food irradiation in Innovative Food Science \& Emerging Technologies, Elsevier, 2009. 
5. Comitê Gestor Da Internet No Brasil; TIC Domicílios e Empresas 2011 - Pesquisa sobre o Uso das Tecnologias de Informação e Comunicação no Brasil.

http://www.cetic.br/media/docs/publicacoes/2/tic-domicilios-e-empresas-2011.pdf (2012)

6. Comitê Gestor Da Internet No Brasil; TIC Domicílios 2014 - Pesquisa sobre o Uso das Tecnologias de Informação e Comunicação nos Domicílios Brasileiros.

http://www.cgi.br/media/docs/publicacoes/2/TIC_Domicilios_2014_livro_eletronico.pdf (2015)

7. Comitê Gestor Da Internet No Brasil; TIC Educação 2011 - Pesquisa sobre o uso das Tecnologias da Informação e Comunicação nas escolas brasileiras.

http://www.cetic.br/media/docs/publicacoes/2/tic-educacao-2011.pdf (2012)

8. Comitê Gestor Da Internet No Brasil; TIC Educação 2014 - Pesquisa sobre o uso das Tecnologias da Informação e Comunicação nas escolas brasileiras.

http://www.cetic.br/media/docs/publicacoes/2/TIC_Educacao_2014_livro_eletronico.pdf (2015)

9. Diretrizes e bases da educação nacional, Lei no 9.394, de 20 de dezembro de 1996, Brazil http:// www.planalto.gov.br/ccivil_03/leis/L9394.htm (2013).

10. Diretrizes Curriculares Nacionais para Educação Básica, Ministério da Educação, Secretaria de Educação Básica, Brazil. http://portal.mec.gov.br/docman/julho-2013pdf/13677-diretrizes-educacao-basica-2013-pdf/file (2013)

11. United Nations Educational, Scientific and Cultural Organization (UNESCO) ; Overview of Information Literacy Resources Worldwide, http://unesdoc.unesco.org/images/0021/002196/219667e.pdf (2013)

12. Levy, D., Villavicencio, A. Web-Based Tools to Increase Public Understanding of Nuclear Technology and Food Irradiation. World Academy of Science, Engineering and Technology, International Science Index 121, International Journal of Social, Behavioral, Educational, Economic, Business and Industrial Engineering, 11(1), 70 - 74, 2017.

13. Arvanitoyannis, I.S. Irradiation of Food Commodities: Techniques, Applications, Detection, Legislation, Safety and Consumer Opinion, Academic Press, Oxford Edition, 2010. 